\title{
Infection of pregnant mice with Listeria monocytogenes induces fetal bradycardia
}

\author{
Jonathan Hardy ${ }^{1}$ Brittani Kirkendoll2 ${ }^{2}$, Hui Zhao' ${ }^{1}$ Laura Pisani ${ }^{3}$, Richard Luong ${ }^{4}$, Alexandra Switzer ${ }^{5}$, Michael V. McConnell ${ }^{6}$ and \\ Christopher H. Contag ${ }^{1,3,5}$
}

INTRODUCTION: Listeriosis is one of the most lethal bacterial diseases for fetuses and infants. However, pregnant women who get infected with Listeria may experience only mild symptoms, making the diagnosis difficult, even when the fetus is fatally infected.

METHODS: To reveal features of this infection, we conducted a multimodality imaging study of Listeria-induced miscarriage, using a pregnant mouse model. In this model, fetal morbidity and mortality can be observed in utero, noninvasively, and the timing and extent of infection can be carefully controlled. By employing in vivo bioluminescence imaging (BLI), perinatal infections were localized over time such that a correlation of infection to outcome could be determined without the need to kill the animal subject. The morbidity and viability of fetuses were assessed with ultrasound, and fetal morphology was imaged using magnetic resonance imaging (MRI).

RESULTS: The ultrasound revealed sustained fetal bradycardia, the slowing of the fetal heartbeat, in infected fetuses, with an association between slowed fetal heart rate and strong bioluminescent signal.

DISCUSSION: Uninfected fetuses showing no bioluminescent signal in the same uterine horn exhibited normal heartbeats. Thus, fetal bradycardia during infection was localized to the infected fetus and was not systemic or disseminated.

$\mathrm{M}$ iscarriage (spontaneous abortion) affects millions of women each year. Although causes of miscarriage such as physical trauma can be determined with relative certainty in some cases, most miscarriages occur without any known etiology. Post facto determination of the cause of a given miscarriage, although important, cannot address mechanisms or means of prevention as directly as experimental investigation. Several animal models of miscarriage have been developed $(1,2)$, but this event is not always predictable even in a laboratory setting. The complex interaction between mother and fetus represents a challenging research problem, and the inability to obtain early data from human miscarriages has greatly impeded our knowledge of this tragic event. Animal models are currently the best hope for understanding the causes and mechanisms of fetal loss. However, most animal models of miscarriage suffer from the inability to both study the mechanism and simultaneously observe the outcome; this information must be obtained from different animals. Therefore, the results are correlative, and dynamic changes are not represented in data derived from subjects killed at selected time points. Live animal, whole-body imaging tools can noninvasively reveal dynamic processes over time, with molecular and cellular specificity. In this manner, pregnant animal subjects can be studied without interfering with the outcome of the pregnancy.

A variety of tools for noninvasive imaging of live animals are available; these offer new opportunities for the analysis of animal models of miscarriage. We used a multimodal imaging approach to study miscarriage associated with listeriosis. The bacterial pathogen Listeria monocytogenes causes abortion, stillbirth, and neonatal meningitis and sepsis in humans $(3,4)$. Pregnant women are 20 times more likely to contract this food-borne illness than nonpregnant individuals, and a woman may exhibit only mild symptoms of listeriosis even when the fetus is killed by the infection. The murine model of perinatal listeriosis is well studied $(5,6)$ and represents an excellent model for the application of molecular imaging techniques to characterize miscarriage in a highly controlled manner. We have employed in vivo bioluminescence imaging (BLI) to track L. monocytogenes in pregnant mice, providing a method of detecting the cause of the miscarriage over time without interfering with the outcome. Magnetic resonance imaging (MRI) was used to image fetal morphology as the infection progressed, and ultrasound was used to assess fetal viability by monitoring fetal heartbeats as the infection progressed. The process of fetal resorption was observed when pregnant animals were infected on embryonic gestational day 8 (E8). Abortion and stillbirth occurred upon infection on E14. Subsequent MRI showed that no resorption occurred on $\mathrm{E} 17,24 \mathrm{~h}$ prior to the fetuses being aborted. Infected fetuses exhibited slowed heartbeats as measured by ultrasound when the BLI signals were strongest, whereas within the same pregnant mouse, fetuses with low BLI signals had heart rates indistinguishable from uninfected controls. These data suggest that a localized effect, such as placental malfunction, causes the

'Department of Pediatrics, Stanford University School of Medicine, Stanford, California; ${ }^{2}$ School of Veterinary Medicine, Tuskegee University, Tuskegee, Alabama; ${ }^{3}$ Department of Radiology, Stanford University School of Medicine, Stanford, California; ${ }^{4}$ Department of Comparative Medicine, Stanford University School of Medicine, Stanford, California; ${ }^{5}$ Department of Microbiology and Immunology, Stanford University School of Medicine, Stanford, California; ${ }^{6}$ Department of Cardiovascular Medicine, Stanford University School of Medicine, Stanford, California. Correspondence: Jonathan Hardy (jonhardy@stanford.edu) 
bradycardia, rather than a systemic process common to all the fetuses. Together, the three complementary imaging modalities offered an unprecedented window into the course of miscarriage, one that can be exploited for the study of many other causes of this pervasive problem.

\section{RESULTS}

\section{BLI of Transplacental Infection}

The bioluminescent $L$. monocytogenes strain $2 \mathrm{C}$ was injected into pregnant CD1 mice intravenously at selected days of gestation (embryonic gestational days E8, E11, E12, or E14; the gestational period of a mouse is $20 \mathrm{~d}$ ). Although humans acquire listeriosis orally from contaminated foods, the intravenous route in mice is well studied and highly reproducible. We selected outbred CD1 mice to better model those human infections in which the mother does not become seriously ill. Normally, these mice are exceptional breeders and produce up to 18 pups per litter, and we have never observed abortion or stillbirth in uninfected CD1 animals (data not shown). Pregnant CD1 mice infected with $5 \times 10^{5}$ colony-forming units (CFU) of L. monocytogenes strain $2 \mathrm{C}$ on E14 exhibit signals indicative of previously well-described transplacental and fetal infections (7-9). One such animal is shown in Figure 1a. BLI signals from areas suggesting placental and/ or fetal infection in this animal were observed, and three fetuses were stillborn (Figure 1b). BLI signals from stillborn pups were assumed to be superficial remnant bacteria, because dead animals
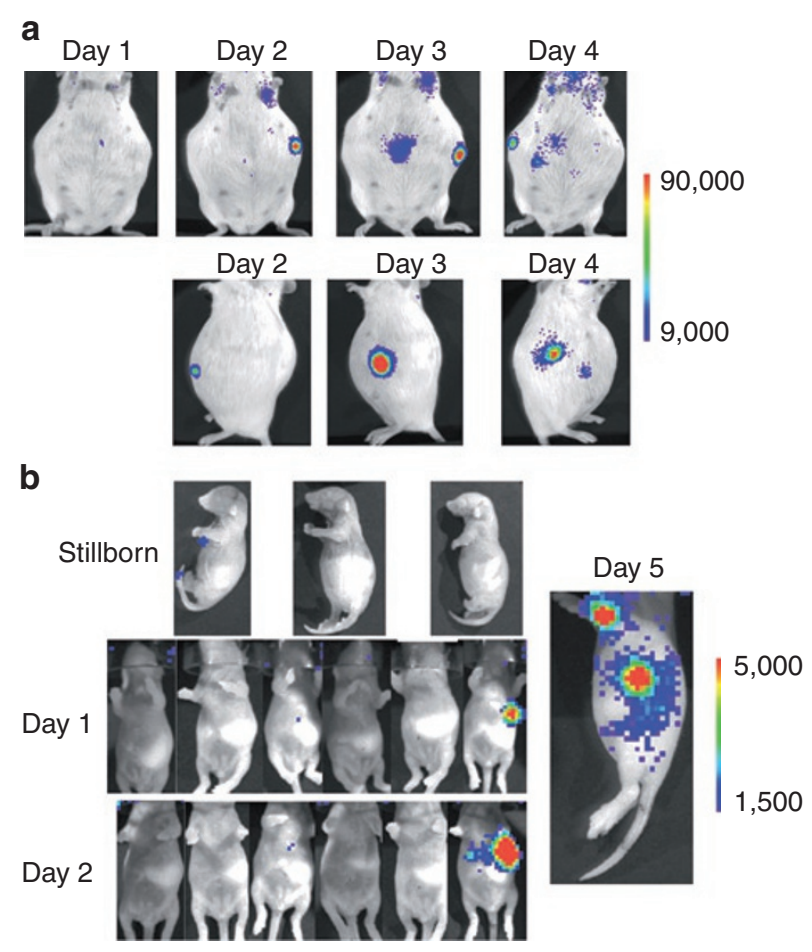

Figure 1. Bioluminescence imaging (BLI) of listeriosis in pregnant mice. Pregnant CD1 mice were infected intravenously with $5 \times 10^{5} \mathrm{CFU}$ (colony-forming units) of bioluminescent Listeria on gestational day 14 (E14). The mice were imaged each day thereafter until birth. (a) BLI of a representative pregnant animal. Ventral (above) and lateral (below) images are shown. (b) BLI of the progeny. Bioluminescence is indicated in photons $/ \mathrm{s} / \mathrm{cm}^{2} / \mathrm{str}$ using the false-color scale shown. rapidly lose BLI signals due to anoxia. Because the precise time of stillbirth was not known, methods such as BLI and histopathology were compromised. Only six pups were born alive to this mouse, an unusually low number for CD1 mice. In addition, BLI signal was observed in one of the live-born pups, and the signal persisted in the animal for $5 \mathrm{~d}$ before it was cleared (Figure $1 \mathrm{~b}$ ). This pup never exhibited signs of severe listeriosis such as matted fur, lethargy, or conjunctivitis. Clearance was expected for most infected progeny, as CD1 mice are highly resistant.

We anticipated different outcomes with different infectious doses and to test this, we infected pregnant animals on E14 using three doses of strain $2 \mathrm{C}$ in three different groups of mice (Figure 2a). At a dose of $1 \times 10^{5} \mathrm{CFU}$, no discernable illness was observed in the mothers, and normal numbers of pups were born, with no stillbirths. Some animals exhibited BLI signals from this dose, but even in the presence of signal the pregnancies appeared to be unaffected, as they proceeded identically to uninfected controls. At a dose of $5 \times 10^{5} \mathrm{CFU}$, again no discernable symptoms were evident in the mother. However, signals were much higher in this group than in the lower dose. The focal signal observed in the lower thorax originated from the gallbladder. L. monocytogenes replicates in the lumen of this organ after either intravenous or oral infection (10). Miscarriage occurred in some animals in the $5 \times 10^{5} \mathrm{CFU}$ group on day 4 postinfection (at E18). Often when miscarriage occurred, BLI indicated the strongest signals emanating from the placentas rather than the fetuses (Figure 2b,c). Histology of the placenta in Figure 2c (Supplementary Figure S1 online) revealed normal murine placental microarchitecture, including recognizable labyrinth, trophospongium (junctional zone), and decidium (decidual basalis). There was no evidence for acute coagulation or lytic necrosis; hemorrhage; thrombosis; or inflammation (including neutrophils or macrophages). Examination of Twort's Gramstained section failed to reveal any observable Gram-positive or Gram-negative organisms. These data suggest that the infection did not result in gross tissue degradation. Signals of the magnitude shown in Figure 2b,c generally represent infection by no more than $1 \times 10^{5} \mathrm{CFU}$. However, ex vivo tissues exhibit nonlinear BLI/CFU relationships because of variable oxygen exposure. At the highest dose of $1 \times 10^{6} \mathrm{CFU}$, very strong BLI signals from the livers of all the pregnant dams were detected on day 3 postinfection, representing more than $1 \times 10^{7} \mathrm{CFU}$ in that organ. The dams were gravely morbid on this day, necessitating euthanasia. When the uterine horns and fetuses were excised postmortem from animals in the high-dose group, signals were detected from the horns and from the fetuses (Figure 2d), indicating that at the high dose, transplacental infection was indeed observed, and that the high signals from the liver may have obscured those of the placenta and fetus in some animals.

\section{MRI of Fetal Morphology}

MRI of infected fetuses revealed no discernable morphological abnormalities at E16 when the infection was initiated on E14, even in animals displaying high bioluminescent signals (Figure 3a). MRI demonstrated that on the day before abortion, no fetal resorption had occurred. The MRI images shown 


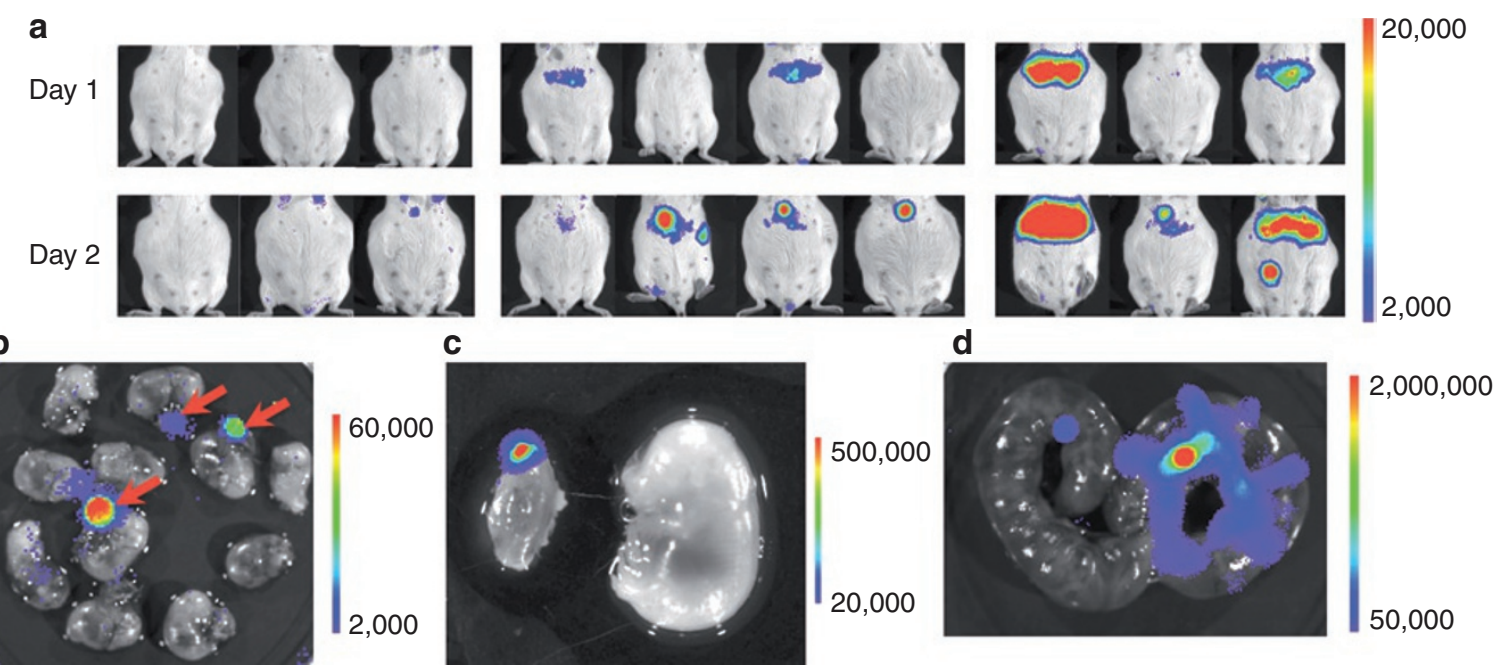

Figure 2. Dose response and signal location. Pregnant CD1 mice were infected with different doses of bioluminescent Listeria and imaged each day thereafter. (a) Mice infected with increasing doses and resulting outcomes are shown on days 1 and 2, after infection. Left panels, $1 \times 10^{5} \mathrm{CFU}$; middle panels, $5 \times 10^{5} \mathrm{CFU}$; right panels, $1 \times 10^{6} \mathrm{CFU}$. (b) Fetuses were dissected from a mouse infected with the highest dose on day 2 and imaged immediately upon dissection. (c) An aborted fetus from the middle dose was imaged after abortion, revealing signal from the placenta (left). (d) Dissected uterine horns from a mouse infected with the highest dose were imaged immediately after dissection. Bioluminescence is indicated in photons $/ \mathrm{s} / \mathrm{cm}^{2} / \mathrm{str}$ using the false-color scale shown. CFU, colony-forming units.

(Figure 3b) were obtained $1 \mathrm{~d}$ before abortion of all the fetuses. MRI provided resolution sufficient to reveal the bones in fetal appendages, as well as brain structures (Figure $3 \mathrm{c}$,d). We have not yet observed morphological abnormalities by MRI, despite resolution of fine anatomical features in late-term fetuses. The structure of the brain and skull, as well as all other morphological details observed, were indistinguishable from uninfected controls. MRI of infected fetuses earlier in gestation was more challenging, as the fetuses are less than $1 \mathrm{~cm}$ in length, and anatomical features that can be observed by MRI are not yet formed. Images from E18 fetuses infected with Listeria revealed that no major morphological differences from uninfected controls were associated with infected fetuses even if they displayed high signals and were aborted the next day.

\section{Assessment of Fetal Viability In Utero}

Ultrasound permits the real-time imaging of dynamic processes in the body such as gallbladder contraction (11) and blood flow (12). We used this modality to assess fetal viability prior to miscarriage, without affecting the process. We assessed fetal viability in the context of listeriosis to determine the timing of fetal death with respect to the course of infection. One such experiment is shown in Figure 4. In this case, BLI signals indicative of fetal infection were observed $3 \mathrm{~d}$ after infection (E17). The animals were then subjected to ultrasound analysis to assess fetal viability by detecting heartbeats. At this time, heartbeats were always detectable from both sides of the animals (data not shown). Imaging was repeated on the subsequent day. On this day, the BLI signal was often more intense on one side (a representative animal is shown). When ultrasound was employed, heartbeats were once again readily detectable on this mouse's left side, as they were on the previous day. However, on the mouse's right side, where the a

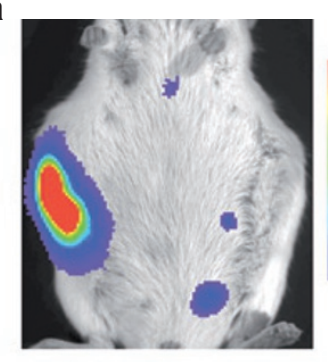

b
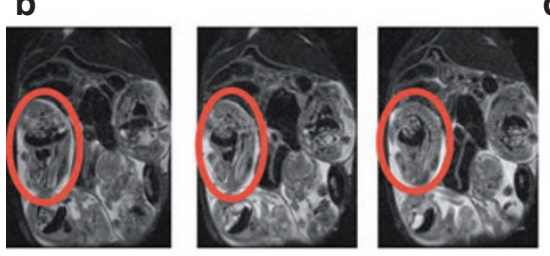

C

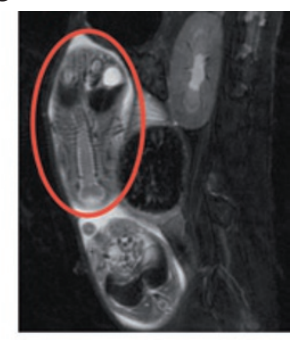

d

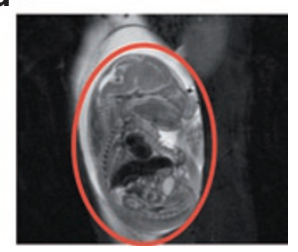

Figure 3. Fetal morphology in infected fetuses assessed by MRI. (a) Pregnant mice infected on embryonic gestational day 14 (E14) with $5 \times 10^{5} \mathrm{CFU}$ of bioluminescent Listeria were imaged with BLI each day thereafter. (b-d) Mice exhibiting strong BLI signals from fetuses were subjected to MRI using a 7 Tesla system (different slices of the same animal shown in (a)). The infected fetus in the animal is circled. (c) and (d) Coronal and sagittal sections, respectively, of an infected fetus on E18. BLI, bioluminescence imaging; CFU, colony-forming units; MRI, magnetic resonance imaging.

BLI signal was more intense, no fetal heartbeats were detectable (Supplementary Videos S1 and S2 online, http://mips. stanford.edu/downloads/ultrasound/, viewed by entering the name "PedResManuscript" and the password "hardy").

\section{Fetal Resorption Due to Infection on E8}

To assess the effects of infection at earlier times during gestation, mice were infected on E8 with $5 \times 10^{5} \mathrm{CFU}$ of Listeria 


\section{Articles $\mid$ Hardyet al.}

strain $2 \mathrm{C}$. This dose was eventually fatal to the pregnant dams, in contrast to infections initiated on E14. Prior to dams becoming moribund from infection, strong BLI signals were observed from areas corresponding to the location of fetuses, as well as from the livers of the pregnant mice (Figure 5a). Ultrasound revealed that infected fetuses were resorbed (Figure 5b). At a lower dose of $1 \times 10^{5} \mathrm{CFU}$, resorption or abortion occurred, and some of the pups born at term were stillbirths. When resorbed fetuses were observed by ultrasound, subsequent

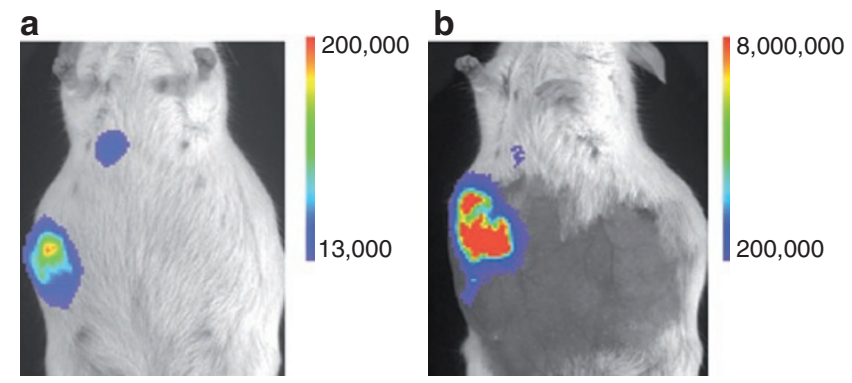

Figure 4. Ultrasound of fetal heartbeats. Pregnant $C D 1$ mice were infected intravenously with $5 \times 10^{5} \mathrm{CFU}$ of bioluminescent Listeria on embryonic gestational day 14 (E14) and imaged each day thereafter. BLI images sometimes showed greater signal on one side of the animal than on the other. Ultrasound performed on day 3 postinfection (a) revealed active fetal heartbeats on both sides of the animal (data not shown). However, on day 4 postinfection, (b) no heartbeats were detectable from the fetuses on the mouse's right side (Supplementary Video S1, http://mips.stanford.edu/ downloads/ultrasound/, viewed by entering the name "PedResManuscript" and the password "hardy"), whereas heartbeats were detected from the fetuses on the left side of the mouse (Supplementary Video S2, http://mips.stanford.edu/downloads/ultrasound/, viewed by entering the name "PedResManuscript" and the password "hardy"). BLI, bioluminescence imaging; CFU, colony-forming units.

a

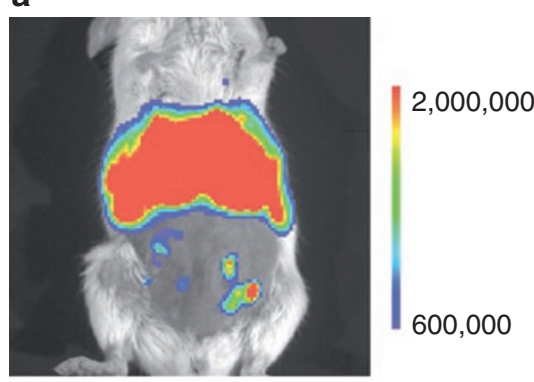

C

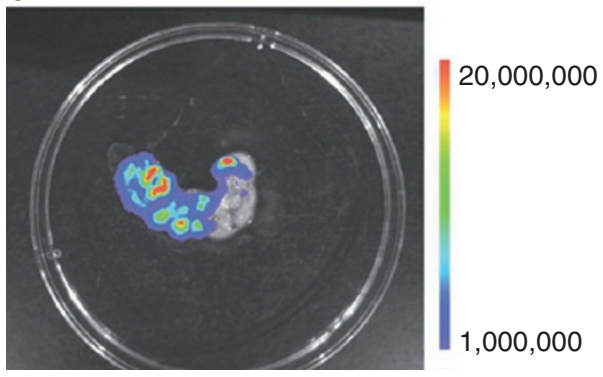

dissection revealed these to be composed of amorphous tissue, and much smaller in mass than a normal fetus on this day of gestation (Figure 5c). Dams with infected, resorbed fetuses often appeared normal and survived despite having extraordinarily high uterine signal levels that, if they had been emanating from the liver, would indicate lethality (Figure 5d). Dams with resorbed fetuses that had weak or absent signals from the liver invariably survived. In addition, animals that aborted fetuses, but also displayed weak or absent signals from the liver, also survived, often despite high signals from the uterus.

\section{Bradycardia Assessed by Ultrasound}

Upon infection, we observed that some fetuses exhibited sustained bradycardia in utero (Figure 6 and Supplementary Videos S3-S7 online; http://mips.stanford.edu/downloads/ ultrasound/, viewed by entering the name "PedResManuscript" and the password "hardy"). This event occurred only in animals in which BLI indicated infection, and only from locations within a pregnant dam that exhibited a BLI signal. The number of heartbeats could be counted for each ultrasound video, and a heart rate could be obtained for each fetus. For example, $6 \mathrm{~d}$ after infection of mice using an inoculum of $1 \times 10^{5} \mathrm{CFU}$ on E12, heart rates of uninfected fetuses were 10-14 beats per 100 frames, whereas infected fetuses were observed to have rates as slow as 2 beats per 100 frames. Irregular heartbeats were also observed in some infected fetuses (Supplementary Video S8 online; http://mips.stanford.edu/downloads/ultrasound/, viewed by entering the name "PedResManuscript" and the password "hardy"). The relation between BLI signal and fetal heart rate exhibited a variable but always negative correlation,
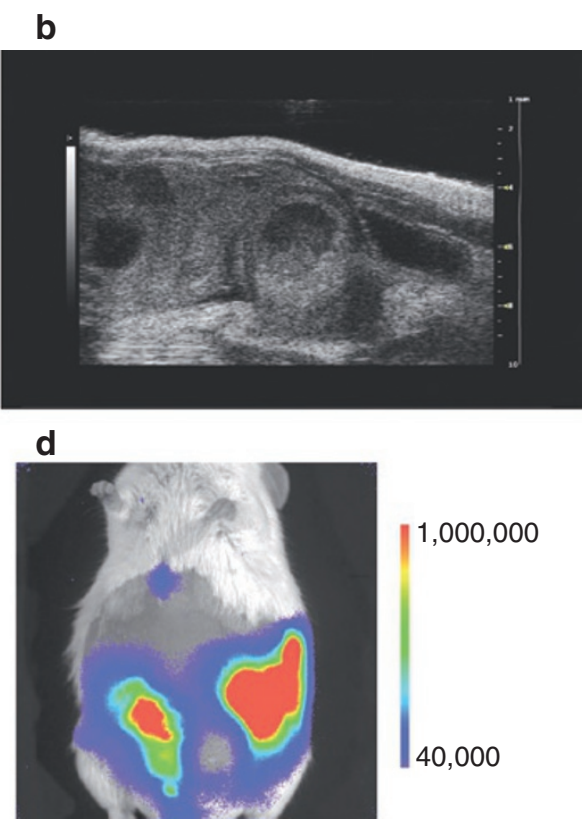

Figure 5. Infection on embryonic gestational day 8 (E8). Pregnant CD1 mice were infected intravenously with $5 \times 10^{5}$ CFU of bioluminescent Listeria on E8 and imaged each day thereafter. (a) This dose produced high signals from the livers of the pregnant dams and necessitated euthanasia in all animals due to severe illness. (b) Before euthanasia, ultrasound revealed fetal resorption, and (c) dissection of the uterine horns showed strong signals from the resorbed fetal tissues. (d) A lower dose of $1 \times 10^{5} \mathrm{CFU}$ on E8 induced resorption (data not shown), but the dams survived despite very high signals. CFU, colony-forming units. 


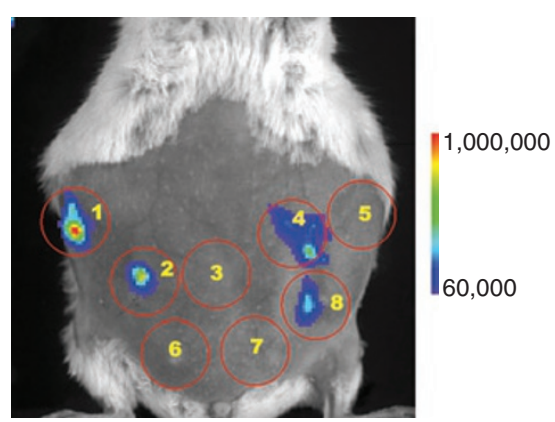

Figure 6. Bradycardia in infected fetuses. Pregnant mice infected with $1 \times 10^{5} \mathrm{CFU}$ on embryonic gestational day 11 were imaged with BLI each day following infection. Ultrasound was performed to compare fetal heart rates with $\mathrm{BLI}$ signals over $1 \mathrm{~cm}^{2}$ circular regions of interest (ROIs). One such animal is shown on day 4 postinfection. ROI $1-1.8 \times 10^{6}$ photons/s/ $\mathrm{cm}^{2} / \mathrm{str}$, heart rate 37.5 beats $/ \mathrm{min}$. ROI $2-1.0 \times 10^{6} \mathrm{photons} / \mathrm{s} / \mathrm{cm}^{2} / \mathrm{str}$, heart rate 62.5 beats $/ \mathrm{min}$. ROI $3-2.0 \times 10^{4}$ photons $/ \mathrm{s} / \mathrm{cm}^{2} / \mathrm{str}$, heart rate 100 beats $/ \mathrm{min}$. ROI $4-1.5 \times 10^{6}$ photons $/ \mathrm{s} / \mathrm{cm}^{2} / \mathrm{str}$, heart rate 37.5 beats/ $\mathrm{min}$. ROI $5-8.2 \times 10^{4}$ photons $/ \mathrm{s} / \mathrm{cm}^{2} / \mathrm{str}$, heart rate 125 beats $/ \mathrm{min}$. ROI $6-2.7 \times 10^{4}$ photons $/ \mathrm{s} / \mathrm{cm}^{2} / \mathrm{str}$, heart rate 150 beats $/ \mathrm{min}$. ROI $7-6.5 \times 10^{4}$ photons $/ \mathrm{s} / \mathrm{cm}^{2} / \mathrm{str}$, heart rate 100 beats $/ \mathrm{min}$. ROI $8-1.4 \times 10^{6}$ photons $/ \mathrm{s} /$ $\mathrm{cm}^{2} / \mathrm{str}$, heart rate 50 beats/min. BLI, bioluminescence imaging; CFU, colony-forming units.

which is depicted graphically in Supplementary Figures S2-S5 online. In fetuses with BLI signals over $1 \times 10^{6}$ photons $/ \mathrm{s} / \mathrm{cm}^{2} /$ str, heart rates $>80$ beats/min were not observed, whereas in fetuses with BLI signals $<1 \times 10^{5}$ photons $/ \mathrm{s} / \mathrm{cm}^{2} / \mathrm{str}$, heart rates below 90 beats/min were never observed. Fetal heart rates in uninfected animals varied even in the same animal, but sustained bradycardia was never observed in the absence of infection (data not shown).

\section{DISCUSSION}

Miscarriage in animal models is a multifactorial process affecting the interaction between mother and fetus. The etiologies and predictors of miscarriage are complex, and, therefore, predictable models need to be developed and evaluated with great care. Models of preterm abortion include the use of toxins or genetic mouse models in which a percentage of fetuses are lost $(1,2)$. The investigation of the details in such models has generally been approached in a manner that precludes the observation of both the cause and effect in the same animal. This limitation, which may appear trivial upon first assessment, is actually highly restrictive when one considers the process as a whole and the need to ascertain its mechanisms. For example, genetic rejection occurs with crosses of $\mathrm{CBA} / \mathrm{J} \times \mathrm{DBA} / 2$ mice (1). These inbred strains, when mated, exhibit spontaneous fetal resorption and miscarriage. Because the mated mice can be compared to those derived from control matings such as $\mathrm{CBA} / \mathrm{J} \times \mathrm{BALB} / \mathrm{c}$, this model is very useful for the study of miscarriage. The outcome of fewer offspring is readily observed when these mice are mated, and the resorption of fetuses can be examined by killing the pregnant animals. These studies have provided important data with regard to miscarriage and its causes, including the interesting role of bacterial lipopolysaccharide (13). However, the tools to predict which fetuses will be resorbed and which will survive have yet to be exploited in this model. By employing live-animal imaging to evaluate miscarriage that results from a known etiology (infection), it becomes possible to assess the temporal features that relate to cause, and to predict a priori which fetuses will be aborted or resorbed. In addition, some of these processes, e.g., fetal resorption, can be observed as they occur, using ultrasound. By correlating morbidity and mortality as assessed by ultrasound to BLI signals as an indicator of infection, mechanisms can be elucidated and the process can be observed without interfering with the outcome. Using this method, we demonstrate that fetal heartbeats are selectively slowed by infection, in a manner that is localized to individual infected fetuses rather than systemic in the pregnant mother. These data may contribute to understanding the mechanisms of fetal morbidity and mortality in this model.

The infection of fetuses by L. monocytogenes is known to occur transplacentally, and fetal trophoblast cells residing in the placenta can be infected by this pathogen $(8,9,14,15)$. Because each murine fetus has its own placenta, individual fetuses can be infected separately. We have observed several fetal pathologies that were correlated with high BLI signals, including bradycardia, irregular heartbeat, and fetal resorption. All of these events occurred only when BLI signals $\geq 1$ $\times 10^{6}$ photons $/ \mathrm{s} / \mathrm{cm}^{2} / \mathrm{str}$ were observed. Uninfected dams showed none of the fetal pathologies observed in infected dams, and uninfected animals delivered from 11 to 18 healthy pups. Aborted fetuses from infected dams sometimes displayed signal only from the placenta (Figure 2), which is consistent with the results of other studies (9). These data suggest that fetal morbidity and mortality could possibly occur via infection of the placenta alone, without invasion of the fetal tissues. However, we were not able to distinguish placental vs. fetal signals by BLI, except by killing the animal. The analysis of aborted fetuses and stillborn pups presents challenges with regard to timing. Perhaps the induction of labor would be useful for future studies.

Mice exhibiting BLI signals $>1 \times 10^{6}$ photons $/ \mathrm{s} / \mathrm{cm}^{2} / \mathrm{str}$ from areas in which fetuses were located invariably aborted all the fetuses. MRI performed late in gestation confirmed that no resorption had occurred in animals that aborted fetuses the next day and showed that infected fetuses were indistinguishable from uninfected fetuses. Some of the pregnant dams exhibiting strong BLI signals from areas corresponding to uterine horns retained such signals for many days postabortion. These mice survived the infection, even when signal levels were extremely high relative to those observed in nonpregnant animals. We have not observed survival of mice exhibiting signals $>1 \times 10^{7}$ photons $/ \mathrm{s} / \mathrm{cm}^{2} /$ str before these experiments and were surprised that dams with infected fetuses exhibiting this magnitude of signal appeared completely asymptomatic and were active. This result may be due to the separation of the fetal compartment from that of the mother, which could prevent infection from spreading once the fetuses are resorbed. Listeria is known to traffic between fetus and mother in guinea pigs (14). 
However, animals that had retained infection after abortion or resorption of the fetuses have not been characterized previously to our knowledge.

MRI of infected fetuses on E18 demonstrated that even fetuses with very high signals were not grossly malformed, nor did they exhibit severe necrosis, which was confirmed by the ultrasound, and revealed that the fetal hearts were normal in appearance. Thus, the bradycardia was not due to fulminant destruction of tissue by the bacteria, but rather some more subtle cause, as is the case with other bacterial infections in humans $(16,17)$. Hearts exhibiting bradycardia were beating normally but slowly and showed no other abnormalities. Contractile function appeared to be preserved. It should be noted that under anesthesia using isoflurane transient bradycardia was observed in uninfected control animals. However, the heart rates always returned to levels indistinguishable from other fetuses within seconds. Listeria monocytogenes secretes a pore-forming toxin, listeriolysin-O, which induces irregular heartbeats when injected into adult rats (18). This protein, which exhibits pleiotropic effects on many cells and tissues, could be the cause of the bradycardia, and experiments are under way to test this hypothesis. These studies suggest that other causes of fetal morbidity and mortality could be examined with similar approaches. However, when the cause is noninfectious, other techniques such as positron emission tomography or single photon emission computed tomography may be necessary to trace the agent inducing fetal pathology.

\section{METHODS}

All mice were housed and maintained according to biosafety level-2 (BSL-2) procedures approved in animal protocol number 12342 by the Administrative Panel for Laboratory Animal Care at Stanford University, using procedures set by the Institutional Animal Care and Use Committees.

\section{In Vivo BLI}

The construction of the bioluminescent L. monocytogenes strain $2 \mathrm{C}$ from the parental L. monocytogenes strain 10403S has been described (10). For a description of the strain, see Supplemental Methods online. For these studies, timed pregnant outbred CD1 mice were purchased from Charles River Laboratories, Wilmington, MA. The mice were infected intravenously. Images were obtained using IVIS 100, 200, and Spectrum systems (Caliper Life Sciences, Alameda, CA) as described by the manufacturer. Isoflurane gas anesthetic was administered at $2 \%$ in oxygen using the Matrix system (Caliper Life Sciences), which enables mild anesthesia, from which the mice recover within $2 \mathrm{~min}$ of removal from the gas. The images were obtained each day following infection using integration times of $1-5 \mathrm{~min}$ at a binning of 2 or 10 pixels for live animals, excised placental tissues, and aborted or stillborn fetuses (indicated in figure legends). Analyses of images were performed using Living Image software (Caliper Life Sciences), and light intensity is displayed as photons per second per square centimeter per steradian (photons $/ \mathrm{s} / \mathrm{cm}^{2} / \mathrm{str}$ ). This calculation ensures that light levels are corrected for exposure and camera settings, providing a measure of absolute light intensity of the source.

\section{MRI}

MRI of infected, pregnant mice was performed on a General Electric (GE; Fairfield CN)-Varian (Agilent Technologies, Santa Clara, CA) pre-product "microSigna 7.0" system. For system description, see Supplementary Methods online. T2-weighted 2D fast-spin echo images with fat saturation were acquired with a repetition time of $3,000 \mathrm{~ms}$, an effective echo time of $71 \mathrm{~ms}$, echo train length of 8 , and 2 averages. Slices were $0.5 \mathrm{~mm}$ thick with a $40 \mathrm{~mm}$ field of view, with a phase field of view of 0.8 , and a $256 \times 256$ acquisition matrix. Anesthesia was administered with $2 \%$ isoflurane in humidified medical-grade oxygen using the Matrix system (Caliper Life Sciences). Physiological monitoring included respiration and temperature feedback for core body temperature maintenance by warm airflow over the supine animal. Artificial tear ointment was applied to the animals eyes to prevent drying.

\section{Ultrasound}

For fetal viability and morbidity studies, the VisualSonics Vevo 2100 instrument (VisualSonics, Toronto, Ontario, Canada) and MS-550D probe were used for morphological analysis and the detection of fetal heartbeats. The Vevo 2100 system easily images the beating hearts of fetuses in the pregnant animal. The mice were first anesthetized via inhalation of isoflurane using the Matrix system (Caliper Life Sciences) and then the abdomen was shaved and treated with depilatory cream to remove the hair. Mice were then placed on the imaging platform of the unit, ultrasound gel was applied to the abdomen, and the mouse was examined with the probe. Morphology of the fetuses was sometimes recorded as still images. Digital video of the fetal heartbeats was recorded according to the instructions of the manufacturer, with each video consisting of exposures of 100 frames in $4.6 \mathrm{~s}$. Heart rate was determined by counting the beats in each video. The entire fetus was scanned if heartbeats were not detected, to verify fetal mortality. Some animals displaying resorbed fetuses were killed and the uterine horns dissected for visual verification and subsequent imaging of the luciferase signals. Ultrasound videos can be viewed at http://mips.stanford.edu/downloads/ultrasound/, by entering the name "PedResManuscript" and the password "hardy" (lowercase).

\section{Histology}

Samples of placenta were placed in $4 \%$ neutral buffered formalin and fixed for at least $24-48 \mathrm{~h}$. The samples were then routinely processed for light microscopic examination of hematoxylin and eosin and Twort's Gram stains.

\section{SUPPLEMENTARY MATERIAL}

Supplementary material is linked to the online version of the paper at http:// www.nature.com/pr

\section{ACKNOWLEDGMENTS}

We are grateful for the technical expertise of Timothy Doyle. We also greatly appreciate the work of James Strommer, who created the ultrasound website, and Pauline Chu, for histology.

\section{STATEMENT OF FINANCIAL SUPPORT}

This project was supported by the Children's Health Research Program, administered by the Lucile Packard Children's Hospital at Stanford, and the Prematurity Research Center at Stanford, administered by the March of Dimes.

\section{REFERENCES}

1. Guimond MJ, Luross JA, Wang B, Terhorst C, Danial S, Croy BA. Absence of natural killer cells during murine pregnancy is associated with reproductive compromise in TgE26 mice. Biol Reprod 1997;56:169-79.

2. Redecha P, van Rooijen N, Torry D, Girardi G. Pravastatin prevents miscarriages in mice: role of tissue factor in placental and fetal injury. Blood 2009; 113:4101-9.

3. Plaza MC, Gilbert-Barness E. Fetal death in utero secondary to Listeria monocytogenes placental infection. Pediatr Pathol Mol Med 2001;20:433-7.

4. Sirry HW, George RH, Whittle MJ. Meningo-encephalitis due to listeria monocytogenes in pregnancy. Br J Obstet Gynaecol 1994;101:1083-4.

5. Abram M, Schlüter D, Vuckovic D, Wraber B, Doric M, Deckert M. Murine model of pregnancy-associated Listeria monocytogenes infection. FEMS Immunol Med Microbiol 2003;35:177-82.

6. Le Monnier A, Join-Lambert OF, Jaubert F, Berche P, Kayal S. Invasion of the placenta during murine listeriosis. Infect Immun 2006;74: 663-72.

7. Abram M, Doric M. Primary Listeria monocytogenes infection in gestating mice. Folia Microbiol (Praha) 1997;42:65-71. 
8. Bakardjiev AI, Stacy BA, Fisher SJ, Portnoy DA. Listeriosis in the pregnant guinea pig: a model of vertical transmission. Infect Immun 2004;72:489-97.

9. Disson O, Grayo S, Huillet E, et al. Conjugated action of two species-specific invasion proteins for fetoplacental listeriosis. Nature 2008;455:1114-8.

10. Hardy J, Francis KP, DeBoer M, Chu P, Gibbs K, Contag CH. Extracellular replication of Listeria monocytogenes in the murine gall bladder. Science 2004;303:851-3.

11. Hardy J, Margolis JJ, Contag CH. Induced biliary excretion of Listeria monocytogenes. Infect Immun 2006;74:1819-27.

12. Sullivan JC, Wang B, Boesen EI, D’Angelo G, Pollock JS, Pollock DM. Novel use of ultrasound to examine regional blood flow in the mouse kidney. Am J Physiol Renal Physiol 2009;297:F228-35.

13. Clark DA, Manuel J, Lee L, Chaouat G, Gorczynski RM, Levy GA. Ecology of danger-dependent cytokine-boosted spontaneous abortion in the CBA $\mathrm{x}$ DBA/2 mouse model. I. Synergistic effect of LPS and (TNF-alpha s+
IFN-gamma) on pregnancy loss. Am J Reprod Immunol 2004;52: 370-8.

14. Bakardjiev AI, Theriot JA, Portnoy DA. Listeria monocytogenes traffics from maternal organs to the placenta and back. PLoS Pathog 2006; 2:e66.

15. Le Monnier A, Autret N, Join-Lambert OF, et al. ActA is required for crossing of the fetoplacental barrier by Listeria monocytogenes. Infect Immun 2007;75:950-7.

16. Hankins GD, Leicht T, Van Hook JW. Prolonged fetal bradycardia secondary to maternal hypothermia in response to urosepsis. Am J Perinatol 1997;14:217-9.

17. Buhimschi C, Weiner CP. Endotoxemia causing fetal bradycardia during urosepsis. Obstet Gynecol 2001;97(5 Pt 2):818-20.

18. Takeda Y, Takeda T, Honda T, Miwatani T. Comparison of bacterial cardiotoxins: thermostable direct hemolysin from Vibrio parahaemolyticus, streptolysin $\mathrm{O}$ and hemolysin from Listeria monocytogenes. Biken J $1978 ; 21: 1-8$. 